\title{
Effects of Efferent Activity on Hair Bundle Mechanics
}

\author{
Chia-Hsi Jessica Lin ${ }^{1}$ and Dolores Bozovic ${ }^{1,2}$ \\ ${ }^{1}$ Department of Physics and Astronomy, and ${ }^{2}$ California NanoSystems Institute, UCLA, Los Angeles, California 90095
}

Hair cells in both the auditory and vestibular systems receive efferent innervation. A number of prior studies have indicated that efferent regulation serves to diminish the overall sensitivity of the auditory system. The efferent pathway is believed to affect the sensitivity and frequency selectivity of the hair cell by modulating its membrane potential. However, its effect on the mechanical response of the hair cell has not been established. We explored how stimulation of the efferent neurons affects the mechanical responsiveness of an individual hair bundle. We tested this effect on in vitro preparations of hair cells in the sacculi of American bullfrogs of both genders. Efferent stimulation routinely resulted in an immediate increase of the frequency of hair bundle spontaneous oscillations for the duration of the stimulus. Enlarging the stimulus amplitude and pulse length, or conversely, decreasing the interpulse interval led to oscillation suppression. Additionally, we tested the effects of efference on the hair bundle response to mechanical stimulation. The receptive field maps of hair cells undergoing efferent actuation demonstrated an overall desensitization with respect to those of unstimulated cells.

Key words: efferent stimulation; frog; hair bundle mechanics; hair cell; sacculus; spontaneous oscillations

\section{Significance Statement}

The efferent system is an important aide for the performance of the auditory system. It has been seen to contribute to sound detection and localization, ototoxicity prevention, and speech comprehension. Although measurements have demonstrated that efference suppresses basilar membrane movement, there is still much unknown about how efferent activity affects hearing mechanics. Here, we explore the mechanical basis for the efferent system's capabilities at the level of the hair bundle. We present optical recordings, receptive field maps, and sensitivity curves that show a hair bundle is desensitized by efferent stimulation. This supports the hypothesis that efferent regulation may be a biological control parameter for tuning the hair bundle's mechanical sensitivity.

\section{Introduction}

The auditory system furnishes us with rich information about the external environment. It contributes crucially to the ability of some species to navigate in space, enables communication with conspecifics, and provides important cues for the avoidance of danger and localization of food sources, among a plethora of applications. These capabilities require not only the sensitivity to detect weak signals as low as $0 \mathrm{~dB}$ (Robinson and McAlpine, 2009; Hudspeth, 2014) but also the ability to extract that information from environments containing multiple competing streams of information with, potentially much louder, background noise. In all of these instances, the auditory system is aided in its tasks by

Received June 5, 2019; revised Jan. 31, 2020; accepted Feb. 9, 2020.

Author contributions: C.-H.J.L. and D.B. designed research; C.-H.J.L. performed research; C.-H.J.L. and D.B. analyzed data; C.-H.J.L. and D.B. wrote the first draft of the paper; C.-H.J.L. and D.B. edited the paper; C.-H.J.L. and D.B. wrote the paper.

This work was supported by National Science Foundation PoLS Grant 1705139 and U.S. Army Research Office Grant W911NF1910179. We thank Dr. Sebastiaan Meenderink for developing the software used for tracking hair bundle movement.

The authors declare no competing financial interests.

Correspondence should be addressed to Dolores Bozovic at bozovic@physics.ucla.edu.

https://doi.org/10.1523/JNEUROSCl.1312-19.2020

Copyright $\odot 2020$ the authors the efferent system (Highstein and Baker, 1985; Highstein, 1991; Micheyl and Collet, 1996; Micheyl et al., 1997; Jamali et al., 2009; Rabbitt and Brownell, 2011; Guinan, 2018).

Furthermore, the delicate machinery of the inner ear is required to sustain very intense signals, sometimes as high as 120 $\mathrm{dB}$ (Hudspeth, 2014), without suffering immediate and irreparable damage. This otoprotective capacity has also been attributed to the efferent system (Handrock and Zeisberg, 1982; Reiter and Liberman, 1995; Maison and Liberman, 2000; Taranda et al., 2009; Boero et al., 2018). Specifically, a number of studies have shown that severing efferent neurons leads to greater levels of ototoxicity. Exposure to moderate or loud sounds was shown to lead to greater hair cell and synaptic damage in animals with lesions to their medial olivocochlear bundle compared with controls (Zheng et al., 2000; Kujawa and Liberman, 2009; Maison et al., 2013), thus establishing an efferent role in protection from acoustic trauma.

Recent research has uncovered even more utilities of the efferent pathway. A set of studies has demonstrated that intact efferent innervation from the lateral olivocochlear and medial olivocochlear nuclei is crucial to maintaining the appropriate balance between both ears (Darrow et al., 2006; Lopez-Poveda et al., 
2016). Since the comparison of interaural signals is essential to accurate sound localization (Seebacher et al., 2019; Zirn et al., 2019), a delicate calibration between the signals received from the two ears must be maintained. Moreover, the efferent architecture has been shown to have a significant function in the "cocktail party effect," which refers to the ability to parse one particular auditory stream from a complex acoustic environment (Giraud et al., 1997; Kumar and Vanaja, 2004; Kim et al., 2006; Andéol et al., 2011; Smith and Keil, 2015; Lopez-Poveda et al., 2016).

As hearing loss constitutes a critical and potentially growing public health problem, understanding the role of the efferent system in controlling hearing sensitivity merits further study. Although a broad literature has established the importance of the efferent system in auditory function (Handrock and Zeisberg, 1982; Micheyl and Collet, 1996; Giraud et al., 1997; Micheyl et al., 1997; Andéol et al., 2011), much less is known about the mechanisms by which it exerts its effect. It has been shown that efferent stimulation suppresses basilar membrane displacement evoked by characteristic frequency tones (Murugasu and Russell, 1996; Russell and Murugasu, 1997; Cooper and Guinan, 2006). Efferent actuation must therefore play a modulatory role in the mechanical response. However, there has been thus far no direct demonstration of the impact of efferent activity on the mechanical responsiveness of the hair bundle.

The hair bundle, an organelle comprised of actin-packed stereovilli arranged in rows of increasing height, sits on the apical side of a hair cell and contains mechanically gated ion channels connected to tip links. Upon deflection of the stereovilli by an incoming sound, tension induced in the tip links causes the opening of transduction channels, enabling the influx of ionic currents. The resulting depolarization of the hair cell leads to the release of neurotransmitters at the afferent synapse (Hudspeth, 1983). The hair bundle's transduction mechanism therefore provides a crucial step in detecting an auditory signal. As damage to this delicate system leads to hearing loss, a protective mechanism that modulates the hair bundle's sensitivity of response would be highly beneficial.

In the current work, we explore the hypothesis that actuating efferent neurons directly affects the mechanical response of a hair bundle. Specifically, we surmise that efferents modulate the bundle's intrinsic active oscillations and lead to a reduction of its sensitivity to mechanical stimulus. To test this hypothesis, we performed experiments on semi-intact preparations that maintained the hair bundles' active motility. Mechanical measurements were combined with efferent stimulation, allowing us to directly probe the efferent impact on a hair cell's mechanical response.

\section{Materials and Methods}

\section{Biological preparation}

Experiments were performed on adult North American bullfrogs (Rana catesbeiana) of either gender. The animals were anesthetized (sodium pentobarbital: $150 \mathrm{mg} / \mathrm{kg}$ ), double-pithed, then decapitated following euthanasia protocols approved by the University of California, Los Angeles Chancellor's Animals Research Committee, in accordance with federal and state regulations. Sacculi were excised from the inner ears of the animals. Saccular maculae were mounted in a two-compartment chamber, which simulated the fluid partitioning of the in vivo environment. The basolateral membrane was bathed in artificial perilymph (in mм as follows: $110 \mathrm{Na}^{+}, 2 \mathrm{~K}^{+}, 1.5 \mathrm{Ca}^{2+}, 113 \mathrm{Cl}^{-}, 3 \mathrm{D}-(+)$-glucose, $1 \mathrm{Na}^{+}$ pyruvate, 1 creatine, 5 HEPES), and the apical surface was immersed in artificial endolymph (in $\mathrm{mm}$ as follows: $2 \mathrm{Na}^{+}, 118 \mathrm{~K}^{+}, 0.25 \mathrm{Ca}^{2+}, 118$ $\mathrm{Cl}^{-}, 3 \mathrm{D}-(+)$-glucose, 5 HEPES). Both solutions were titrated to match the $\mathrm{pH}$ and osmolarity of the physiological conditions in the otic laby- rinth and freshly oxygenated immediately before use. The overlying otolithic membrane was enzymatically dissociated from the epithelium with $50 \mu \mathrm{g} / \mathrm{ml}$ collagenase IA-S (Sigma Millipore) for $8 \mathrm{~min}$ and gently removed. Spontaneous oscillations were observed in hair bundles after the membrane was decoupled and could be sustained for several hours after dissection. For control experiments, $2 \mu \mathrm{M}$ strychnine (Sigma Millipore), $1 \mu \mathrm{M}$ tubocurarine chloride (Tocris Bioscience), or $50 \mu \mathrm{M}$ apamin (Sigma Millipore) was added to the artificial perilymph (Sugai et al., 1992; Guth et al., 1994; Yoshida et al., 2001). The solution in the basal compartment was replaced via a fluid exchange, and measurements were repeated after a 5 min incubation period (Rossi et al., 1980; Prigioni et al., 1983; Murugasu and Russell, 1996).

\section{Imaging and tracking hair bundle motion}

Data acquisition was performed inside an acoustically isolated chamber (Industrial Acoustics) with the microscope setup mounted on a vibration-isolation table (Technical Manufacturing). Preparations were imaged in an upright optical microscope (Olympus BX51WI) with a water-immersion objective (Olympus XLUMPlanFL N, 20×, $1.00 \mathrm{NA}$ ). Images were further magnified (for $\sim 400 \times$ total magnification with camera pixel resolution of $52.1 \mathrm{~nm} / \mathrm{px}$ ) and projected onto a high-speed CMOS camera (Photron FASTCAM SA1.1) recording at 1000 frames per second. Hair bundles were imaged at the focal plane intersecting the tallest row of stereocilia, and a hair bundle's position was derived for displacements toward and away from its kinocilium. For each preparation, multiple hair bundles could be tracked in an FOV. Recordings of bundles within the same epithelium were therefore obtained simultaneously for all stimuli applied. From these recordings, the motion of a hair bundle was tracked with software written in MATLAB (The MathWorks). The position of the hair bundle in each frame was determined by calculating the center of gravity of its intensity profile along a row of pixels. To enhance the signal-to-noise ratio, this calculation was performed with at least 10 rows of adjacent pixels to extract the mean position in each frame. A time-dependent position trace of a hair bundle's movement was obtained by plotting the mean position for each frame of the recording.

\section{Efferent stimulation}

The saccular nerve was pulled into a $0.5-\mathrm{mm}$-diameter silicon tube (Castellano-Muñoz et al., 2010), which was electrically connected to the positive electrode of a bipolar suction electrode (A-M Systems). The reference electrode was placed into the basolateral compartment. The preparations were measured to have resistances in the $1-2 \mathrm{M} \Omega$ range. A linear stimulus isolator (World Precision Instruments A395) provided 10-250 $\mu \mathrm{A}$ of current to the suction electrode, and stimulus protocols were sent to the isolator via LabView (National Instruments). Throughout this manuscript, a pulse train will be described by its "on" (pulse duration) and "off" (interpulse interval) times (Fig. 1B). A typical recording consisted of 2-7 s without efferent stimulus, followed by 5-7 s of efferent activity, and terminating with a return to the control (no efferent stimulus) condition for 7-14 s.

\section{Mechanical stimulation}

A glass fiber $\sim 1 \mu \mathrm{m}$ in diameter was created with a modified microforge to fabricate an additional rod perpendicular to the tip of a borosilicate glass capillary that had been pulled with a micropipette puller (Sutter Instruments). The stiffness and viscous drag coefficient of the glass probe were measured by recording the Brownian motion of the tip of the glass fiber in water and fitting its power spectral density to a Lorentzian function (Howard and Hudspeth, 1987). The stiffness and drag coefficients of the glass probes used in these experiments were 75-150 $\mu \mathrm{N} / \mathrm{m}$ and $100-$ $150 \mathrm{nN} \cdot \mathrm{s} / \mathrm{m}$, respectively. With these specifications, a $10 \mathrm{~nm}$ probe displacement corresponds to an $\sim 1 \mathrm{pN}$ force on the bundle. Probes were mounted on a piezoelectric actuator (Piezosystem Jena PA 4/12) at an angle of $\sim 30$ degrees with respect to the preparation and positioned to attach to either the kinocilium or the tallest row of stereocilia. The probe was micromanipulated to adjoin a single hair bundle, and visual confirmation was used to ensure that the glass fiber was in contact with only one hair bundle (Fig. 1D). Before attachment, the fiber tip was dipped into $5 \mathrm{mg} / \mathrm{ml}$ concanavalin-A, a highly charged polymer, to enhance the 
adhesion. Synchronously with efferent stimulation and video acquisition, $3-50 \mathrm{~Hz}$ sinusoidal signals with amplitudes ranging from 4 to $80 \mathrm{~nm}$ were sent to the piezoelectric amplifier via LabView (National Instruments). Mechanical stimuli of varying frequencies and amplitudes were applied sequentially, without any intervals between the signals. Unless indicated otherwise, the sinusoidal amplitude is reported as the amplitude applied to the base of the probe. Displacements to the base of the probe generated lateral sinusoidal displacements of the hair bundle, mimicking the mechanical stimulation produced by sound in vivo.

\section{Data analysis}

Determination of spontaneous oscillation frequency, amplitude, and open probability. In this manuscript, positive displacement in the traces corresponds to motion toward the kinocilium and leading to channel opening, consistent with the standard convention in the literature. Before analysis, the effects of slow drift in the epithelium were subtracted from hair bundle position traces. Traces of hair bundles that underwent only efferent stimulation were split into three sections corresponding to recordings obtained before, during, and after the application of the efferent stimulus and analyzed separately. Each section was established as being oscillatory only if its Hartigans' dip statistic was $>0.01$ and the associated $p$ value was $<0.001$, an indication of multimodality in the position distribution (Hartigan and Hartigan, 1985; Salvi et al., 2015, 2016). Our analysis only included those position traces for which all three divisions were classified as oscillatory.

Several parameters were extracted from the position traces using software implemented in Python. The histogram of the bundle position was calculated for each of the three sections, and a kernel density estimate was applied to obtain a continuous probability distribution. The two local maxima were identified in each distribution, and the minimum between these peaks was selected to be the threshold between the open channel and closed channel states. The amplitude of the spontaneous oscillation was defined to be half of the distance between the two peaks. The mean open probability was obtained by computing the area under the position distribution plot with a position value greater than the threshold, divided by the area under the full probability density function. Using the aforementioned open channel threshold, any excursion of the bundle beyond the threshold was designated as a positive transition to open transduction channels. The period between two consecutive, positive threshold crossings defined the instantaneous period of one cycle, from which we acquired the instantaneous frequency. All instantaneous frequencies tabulated within a section were averaged to obtain the mean oscillation frequency. Each hair cell was treated as one element of an ensemble. Thus, parameters that were extracted from cells undergoing the same stimulus conditions were averaged together.

Receptive field maps and sensitivity curves. Receptive field maps were measured by calculating the phase-locked component of a hair bundle's response to sinusoidal deflection over a range of mechanical drive frequencies. The applied frequency spanned the range $3-50 \mathrm{~Hz}$ in $2 \mathrm{~Hz}$ increments. Ten cycles were presented for each stimulus frequency. The full sweep was administered at stimulus amplitudes varying from 4 to 80 $\mathrm{nm}$. For a particular stimulus frequency and stimulus amplitude, the corresponding bundle position trace was divided into 10 segments and averaged. A single sine wave with the corresponding stimulus frequency was fit to the averaged response, and its amplitude was extracted to obtain the phase-locked component.

At a specific stimulus amplitude, a hair bundle's sensitivity, $\chi\left(\omega_{\mathrm{S}}\right)$, was computed from the following:

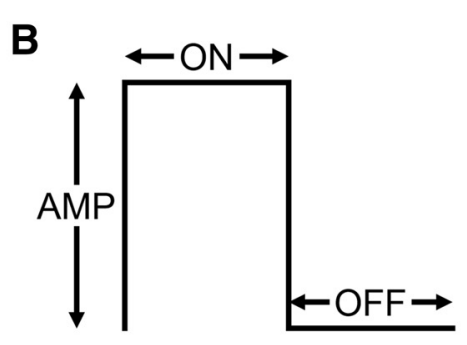

D

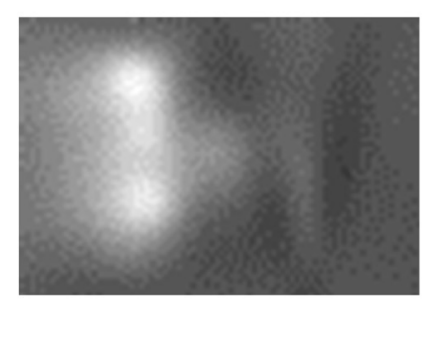

\section{(1)}

High-Speed

Camera

Endolymph

Sacculus
Sth $\left\|_{\boldsymbol{h}}\right\|_{\boldsymbol{h}} \|_{\boldsymbol{r}}$

ll.
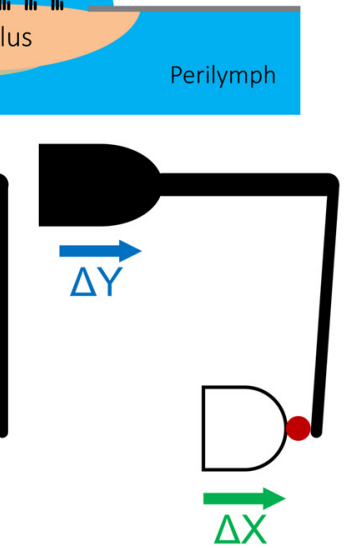

Figure 1. $\quad \boldsymbol{A}$, The efferent neurons of the bullfrog sacculus were stimulated by inserting the eighth cranial nerve into a silicon isolar A two-compartment setup was used to mimic the ionic concentrations for the natural fluid environment of the sacculus.

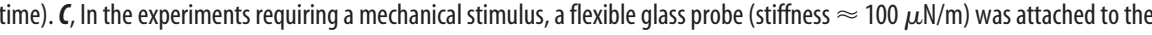
nocilium (red dot) of the hair bundle (HB). A displacement at the base of the probe caused a displacement in the position of the hair bundle. $D$, Bright field image of a single hair bundle with a glass fiber attached to the kinocilium.

$$
\chi\left(\omega_{S}\right)=\frac{A_{p l}\left(\omega_{S}\right)}{f\left(\omega_{S}\right)}
$$

where $\omega_{\mathrm{S}}$ is the stimulus frequency, $A_{p l}\left(\omega_{S}\right)$ is the phase-locked amplitude of the bundle oscillation, and the force $f\left(\omega_{\mathrm{S}}\right)$ is given by the following:

$$
f\left(\omega_{S}\right)=\left(K_{F}+i \omega_{S} \xi\right) A_{S},
$$

where $K_{F}$ is the glass fiber's stiffness, $\xi$ is its viscous drag coefficient, and $A_{S}$ is the stimulus amplitude (Martin and Hudspeth, 2001).

\section{Statistical analysis}

All statistical analyses were performed using Python. The difference between a control and a condition for a population was assessed using a one-tailed paired $t$ test. The differences were considered significant if $p<$ 0.01 . The sample size for an experiment was considered sufficient if the resulting $p$ values met the requirement for statistical significance. The sample size was defined to be the number of hair cells measured, as the focus of this study is on the effect of efference on individual hair cells, which have been mechanically decoupled by removing the otolithic membrane. The innate activity of the hair bundles within a sacculus has previously been shown to be uncorrelated (Ramunno-Johnson et al., 2009), as there is no known transverse coupling between hair bundles or retrograde effects on the efferent neurons.

\section{Results}

\section{Efferent stimulation affects hair bundle} spontaneous oscillations

We recorded the motion of spontaneously oscillating hair bundles from the sensory epithelium of the American bullfrog sacculus before, during, and after electrically stimulating the saccular nerve. There was no predetermined location within the macula from which hair bundles were chosen, as all the hair cells in the bullfrog's sacculus have been shown to be highly innervated by efferent fibers (Castellano-Muñoz et al., 2010). When the efferents were actuated by application of a current to the nerve, hair bundles oscillated with a noticeably higher frequency than they did without efferent stimulation (Fig. 2A). This change occurred 
A

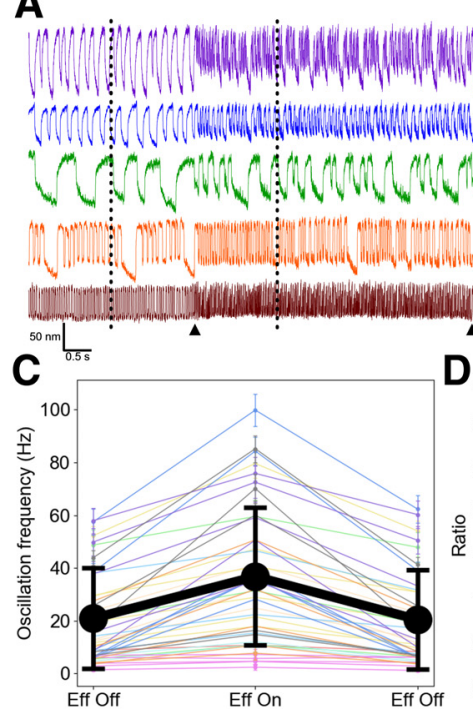

B

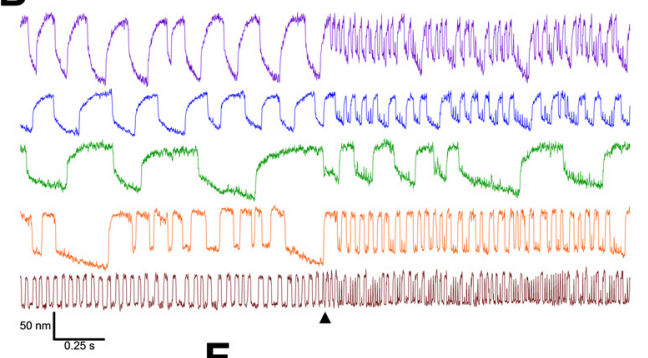

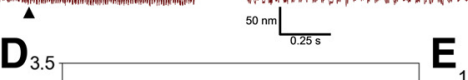

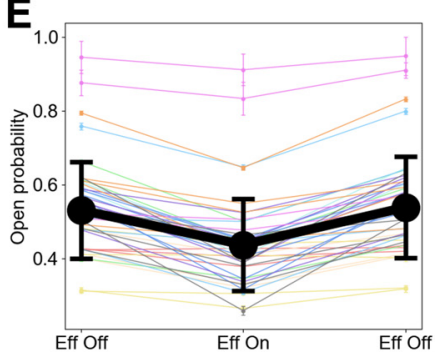

Figure 2. The frequency of spontaneous oscillations increased immediately upon the onset of the efferent stimulus. $\boldsymbol{A}$, Spontaneous oscillations before, during, and after efferent modulation (pulse train: $200 \mu \mathrm{A}, 1$ ms "on," 10 ms "off") are displayed for five representative hair bundles procured from three different sacculi, shown in different colors, and offset for clarity. The efferent stimulus was in effect for the duration occurring between the two black triangles illustrated below the hair bundle position traces. $\boldsymbol{B}, A$ zoom-in of the region between the dashed lines in $\boldsymbol{A}$ shows that the stimulus exerts an effect rapidly upon onset. $\boldsymbol{C}, 0$ n average, hair bundles demonstrate an increase in oscillation frequency during stimulation and a return to their original spontaneous oscillation frequency upon cessation of the stimulus. Individual frequency data points are plotted in various colors for hair bundles undergoing the pulse train efferent stimulation described in $\boldsymbol{A}$. Hair bundles from the same sacculus are plotted in the same color. The corresponding error bars were calculated from the SDs of the instantaneous frequencies given by the oscillation cycles during the efferent stimulus. The colored data points in each of the three sections were averaged over 47 hair cells, and the averages are displayed by the large black data points. Black error bars were calculated from the SDs of the individual bundle frequencies. The data shown were taken from recordings of 47 hair bundles obtained from 11 sacculi. $\boldsymbol{E}$, Hair bundles display, on average, a decrease in open probability during efferent activity, and a return to the original open probability after stimulus. The colored data points represent the open probabilities of individual hair bundles, and their error bars were obtained by calculating the integrated squared error of the probability density function. Hair bundles from the same sacculus are plotted in the same color. For each stimulus condition, the averages were calculated over 47 hair cells and are plotted in black. Error bars were calculated from the SDs of the individual bundle open probabilities. The data shown were obtained from the same hair bundles in C. D, The ratio of the frequency during efferent modulation (F2) to the frequency prestimulus (F1), as well as the ratio of the frequency after stimulus (F3) to F1, were calculated for every hair bundle shown in $C$. The means of the averages $\langle F 2 / F 1\rangle$ and $\langle F 3 / F 1\rangle$, computed over 47 cells, are plotted in blue. Analogous open probability ratios ( $02 / 01\rangle$ and $\langle 03 / 01\rangle$ ) were computed, averaged and are shown in orange. Error bars were derived from the SDs of the individual ratios. $\boldsymbol{C}-\boldsymbol{E}$, All cells were stimulated with the same pulse train parameters detailed in $\boldsymbol{A}$.

immediately after the onset of the stimulus (Fig. $2 B$ ) and ceased upon its termination. The temporal profile of the oscillation also changed upon the stimulus onset, showing smaller and sharper excursions of the bundle. Quantitative analysis of the spontaneous oscillation frequencies of 47 hair cells confirmed the observed change and return to original value (Fig. 2C). The oscillation frequencies of the hair bundles increased by an average of $15.86 \pm$ $13.53 \mathrm{~Hz}$ (one-tailed paired $t$ test, $t_{(46)}=8.04, p=1.31 \times 10^{-10}$ ). Moreover, analyzing the open probabilities of these same hair cells revealed that the open probability declined during efferent activity and recovered upon cessation of the stimulus (Fig. 2E). The open probabilities of the hair bundles decreased by an average of $-0.09 \pm 0.07$ (one-tailed paired $t$ test, $t_{(46)}=-9.11, p=$ $\left.3.66 \times 10^{-12}\right)$. While each hair bundle's frequency and open probability increased and decreased, respectively, by a different factor, the same trend was observed across all of the cells measured and by the average behavior of the sampled cells. Furthermore, by computing the individual ratios of the frequency during stimulation (F2) to the frequency before stimulation (F1) and averaging over the cells, we found the oscillation frequencies increased by a mean factor, $\langle\mathrm{F} 2 / \mathrm{F} 1\rangle$, of $2.20 \pm 0.84$ (one-tailed paired $t$ test, $t_{(46)}=6.36, p=4.21 \times 10^{-8}$; seen in Fig. $\left.2 D\right)$. A similar calculation was performed for the open probability, and it yielded a mean ratio, $\langle\mathrm{O} 2 / \mathrm{O} 1\rangle$, of $0.83 \pm 0.13$ (one-tailed paired $t$ test, $t_{(46)}=-9.35, p=1.67 \times 10^{-12}$ ).

We confirmed that the efferent synaptic activity was crucial for the observed effect by introducing $2 \mu \mathrm{M}$ strychnine or $1 \mu \mathrm{M}$ D-tubocurarine into the basolateral compartment. Both strychnine and D-tubocurarine block ACh from binding with nicotinic receptors ( $\mathrm{nAChRs)}$ in the hair cell soma and thereby abolish any effect that the efferent synapses exert on the cell (Sugai et al., 1992; Guth et al., 1994). Upon the addition of either strychnine or D-tubocurarine into the perilymph solution, the hair bundles' oscillation profiles remained unchanged during the efferent pulse train (200 $\mu \mathrm{A}, 1 \mathrm{~ms}$ “on," $10 \mathrm{~ms}$ "off") stimulus (Fig. 3 A,D). This was markedly different from the increased frequencies measured in standard perilymph. We observed a full recovery of the original response to efferent actuation in the innate oscillation frequencies of the hair bundles (Fig. $3 B, E$ ) after completing a rinse back to standard perilymph. In standard perilymph, the hair bundles in Figure $3 B$ experienced an average frequency increase of $22.62 \pm 13.84 \mathrm{~Hz}$ (one-tailed paired $t$ test, $t_{(10)}=5.42, p=$ $0.00015)$ during efferent modulation. After the addition of strychnine, the average change in frequency upon efferent stimulation was $0.12 \pm 1.75 \mathrm{~Hz}$ (two-tailed paired $t$ test, $t_{(10)}=0.23$, $p=0.82$ ). In the case of Figure $3 E$, hair bundles immersed in standard perilymph demonstrated, on average, a frequency increase of $19.32 \pm 9.81 \mathrm{~Hz}$ (one-tailed paired $t$ test, $t_{(10)}=6.53$, $p=3.30 \times 10^{-5}$ ) under efferent stimulation. When $\mathrm{D}$ tubocurarine was used to block the nAChRs, the average change in frequency was reduced to $-0.11 \pm 0.81 \mathrm{~Hz}$ (two-tailed paired $t$ test, $t_{(10)}=-0.45, p=0.66$ ). This control was also performed for a step $(200 \mu \mathrm{A})$ stimulus and resulted in the same outcome (Fig. 3C,F), with the effects of efferents fully abolished by the blockers. The control experiments confirmed that our method of electrically stimulating the saccular nerve was exerting its effect on hair bundles by activating the efferent neurons. 

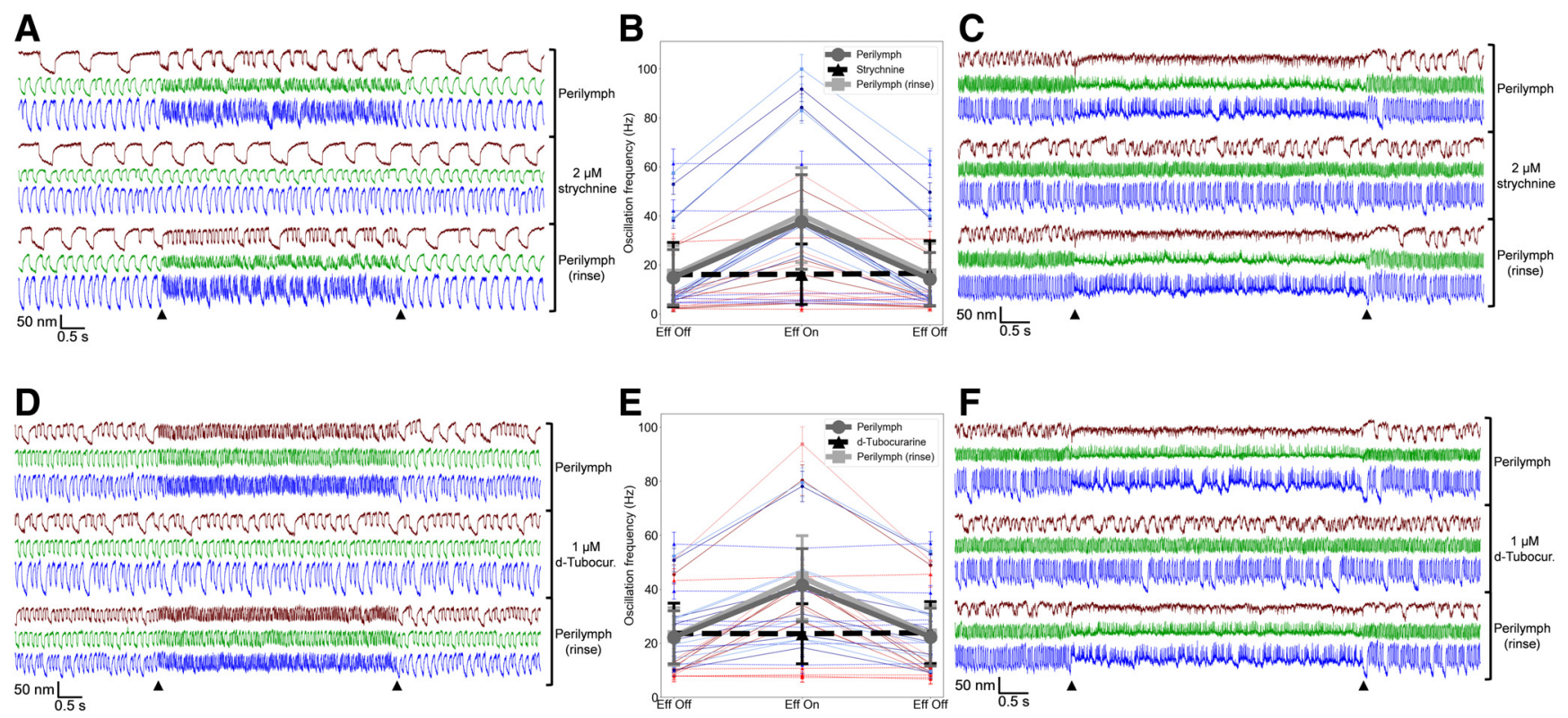

Figure 3. Strychnine and D-tubocurarine, added into the perilymph bath solution, abolish the effects of efferent stimulation. The control was performed for both pulse train $(\boldsymbol{A}, \mathbf{D} ;$ stimulus: 200 $\mu \mathrm{A}, 1 \mathrm{~ms}$ "on," $10 \mathrm{~ms}$ " off") and step ( $\boldsymbol{C}, \boldsymbol{F}$; stimulus: $200 \mu \mathrm{A})$ stimuli, and three different bundles are shown in each subfigure. Upon blockage of the receptors, the efferent stimulus exerts no visible effects on innate bundle motility. The average hair bundle oscillation frequency does not change upon efferent stimulation when strychnine $(\boldsymbol{B})$ or $\mathrm{D}$-tubocurarine $(\boldsymbol{E})$ is introduced into the basal bath solution. Circles, triangles, and squares represent oscillation frequencies in standard perilymph, strychnine or D-tubocurarine perilymph, and standard perilymph (rinse), respectively. $\boldsymbol{B}$ was averaged from 11 bundles ( 2 sacculi). $\boldsymbol{E}$ was averaged from 11 bundles ( 2 sacculi). Hair bundles originating from the same sacculus were plotted in shades of the same color. The effects in $\boldsymbol{C}$ and $\boldsymbol{F}$ were observed in 8 bundles ( 2 sacculi) each. Error bars indicate the SDs of individual hair bundle oscillation frequencies.

\section{Hair bundle's response to efferent modulation is dependent on stimulus parameters}

When an electrical pulse is sent to the presynaptic terminal, the terminal becomes depolarized and releases ACh (Wedemeyer et al., 2018). The ACh binds to $\alpha 9 \alpha 10$ nicotinic receptors (GómezCasati et al., 2005; Ballestero et al., 2011; Guinan, 2011), which enhances the influx of $\mathrm{Ca}^{2+}$ ions into the hair cell soma. The elevated $\mathrm{Ca}^{2+}$ level triggers the opening of $\mathrm{Ca}^{2+}$-activated SK2 $\mathrm{K}^{+}$channels (Blanchet et al., 1996; Oliver et al., 2000; Rohmann et al., 2015), and the efflux of $\mathrm{K}^{+}$hyperpolarizes the cell (Fuchs and Murrow, 1992; Fuchs, 2002; Kong et al., 2008). The extent to which the hair cell becomes hyperpolarized is hence determined by the amount of ACh that is released at the synapses, which is in turn influenced by the applied electrical signal (Taylor and Brown, 1999; Westfall, 2009). We therefore varied the parameters of the efferent stimulus trains and observed the effects of this variation on the hair bundle's spontaneous oscillation profile.

We presented pulse train stimuli via the suction electrode (as described in Materials and Methods), and separately varied the pulse duration ("on" time) and the interval between pulses ("off" time). For the first set of experiments, the amplitude was kept constant at $200 \mu \mathrm{A}$ and the "off" time at $10 \mathrm{~ms}$, with the pulse duration incrementally extended from 0.5 to $50 \mathrm{~ms}$ (Fig. $4 A$ ). Initially, the hair bundles oscillated more rapidly with increasing pulse duration. As the "on" time was further increased, the hair bundles began to show rapid, spike-like excursions and an increasing propensity for the closed channel state. In addition, a slow cumulative offset in the negative direction was observed in the last few traces of Figure $4 A$. This induced negative drift was detected for the majority of the hair bundles measured. Of the hair bundles that exhibited this characteristic ( 9 of the 10 cells recorded across two preparations), the magnitude of the drift positively correlated with the "on" time. At the highest "on" time of $50 \mathrm{~ms}$, the bundles drifted by an average of $55 \pm$ $35 \mathrm{~nm}$ in the negative direction (away from the kinocilium), with some bundles drifting up to $140 \mathrm{~nm}$. All bundles were observed to recover from their negative offset, with recovery from stimuli with longer pulse durations being more protracted. Likewise, the bundles that were seen to oscillate with a lower frequency directly after stimulus were observed to return to their initial spontaneous oscillation frequencies. Subsequently, the interpulse interval was decreased from 40 to $1 \mathrm{~ms}$ while maintaining a constant amplitude of $200 \mu \mathrm{A}$ and a pulse duration of $1 \mathrm{~ms}$ (Fig. 4C,D). Decreasing the "off" time of the pulse train elicited similar results to those observed when increasing the "on" time of the pulse train; the frequency of the bundle oscillations increased as the "off" time decreased.

Last, we investigated the dependence of the hair bundles' oscillation profiles on the amplitude of the applied efferent stimulus. We increased the amplitude of the pulse train ( $3 \mathrm{~ms}$ "on" time and $10 \mathrm{~ms}$ "off" time) from 10 to $250 \mu \mathrm{A}$. The bundle traces shown in Figure $5 \mathrm{~A}$ demonstrate that the rising current amplitude elevates the impact of the efferent activity. A similar outcome was achieved when the experiment was repeated with a current step applied instead of a pulse train (Fig. $5 B$ ). Oscillation suppression was attained more readily with the step stimulus than with the pulse train stimulus. Additionally, half ( 6 of the 12 hair cells recorded across 3 preparations) of the hair bundles were observed to negatively drift during the step stimulus.

To quantify the impact of varying efferent stimuli on the innate motility of hair bundles, we used oscillation analysis software implemented in Python (see Materials and Methods). Three parameters were extracted to characterize the temporal profile of the bundle oscillation: oscillation frequency (Fig. $6 A, D, G$ ), oscillation amplitude (Fig. 6B,E,H), and mean channel opening probability (Fig. $6 C, F, I$ ). The changes induced in the hair bundles' oscillations were measured with changing stimulus pulse 
A

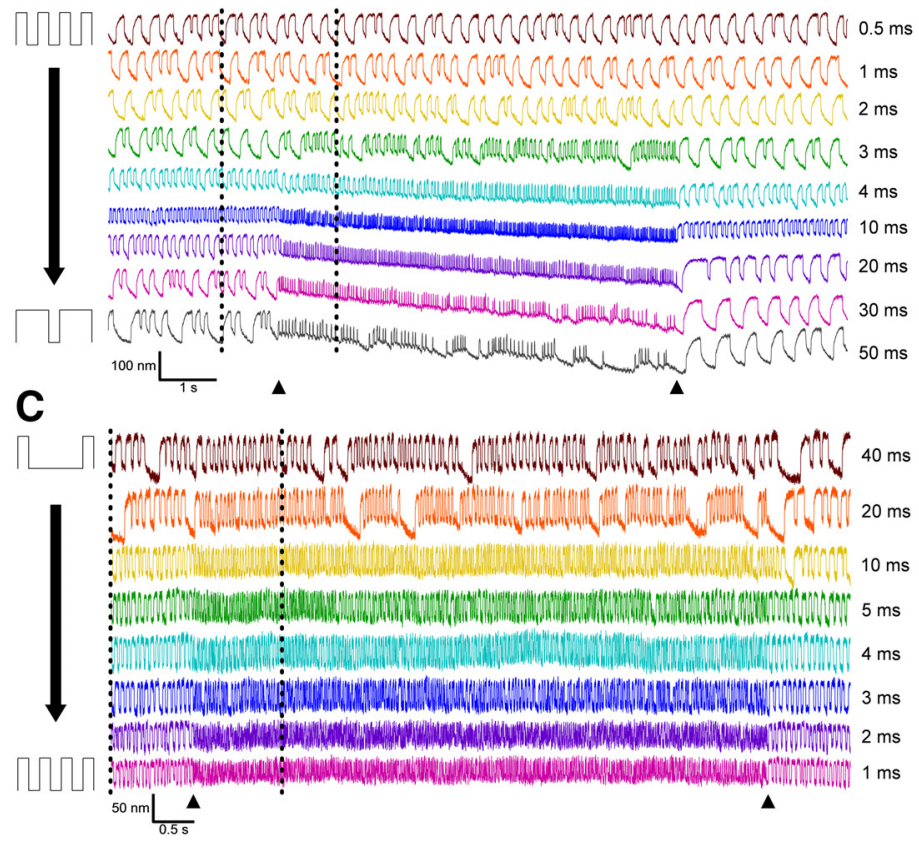

B

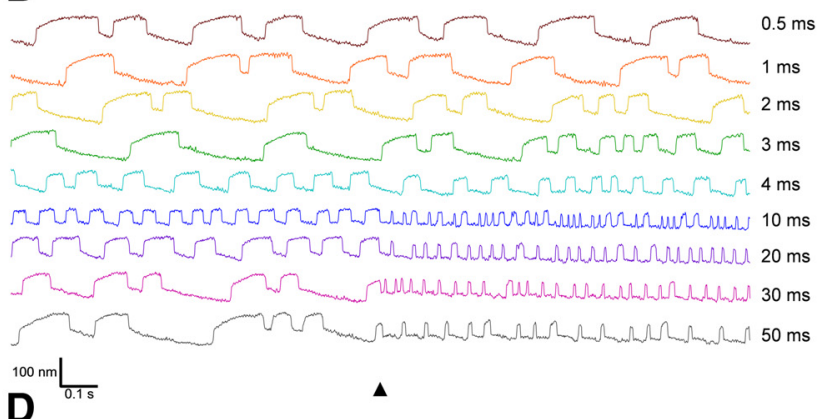

Figure 4. Variations of the pulse train stimulus applied to the saccular nerve resulted in changes to the spontaneous oscillation profile of the hair bundles. The amplitude of the pulse train was kept constant at $200 \mu \mathrm{A}$, whereas the pulse duration ("on" time) and the interval between pulses ("off" time) were separately varied. $\boldsymbol{A}$, The interpulse interval was kept at 10 $\mathrm{ms}$ while the pulse duration was systematically altered. As the pulse duration increased, the oscillation frequency of the bundle correspondingly increased until the bundle began to exhibit short spike-like excursions, seen in the zoom-in $(\boldsymbol{B})$ of the region between the dashed lines in $\boldsymbol{A}$. $\boldsymbol{C}$, The interpulse interval was systematically varied, with the pulse duration held constant at $1 \mathrm{~ms}$. The hair bundle's oscillation frequency increased with decreasing interpulse interval, seen clearly in the zoom-in (D) of the region between the dashed lines in $\boldsymbol{C}$. The efferent stimulus occurred between the two black triangles illustrated below the hair bundle position traces. $A, C$, Left schematics represent the manner in which the pulse train stimulus is being varied and, for purposes of visibility, are not drawn to scale.

A

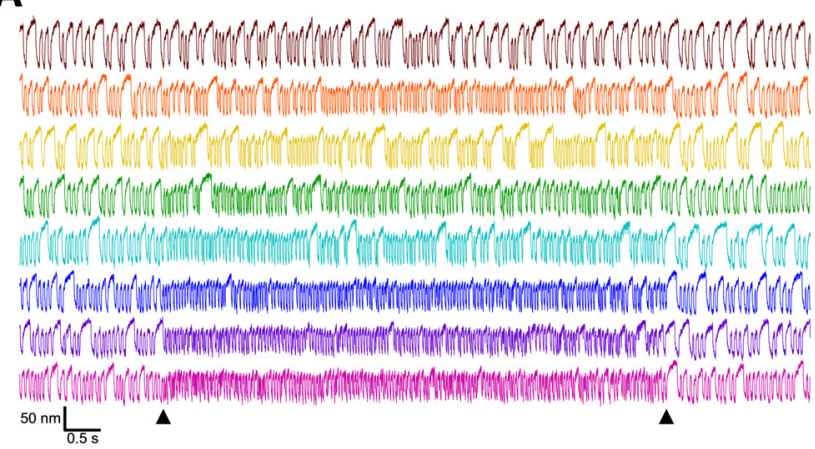

\section{B}

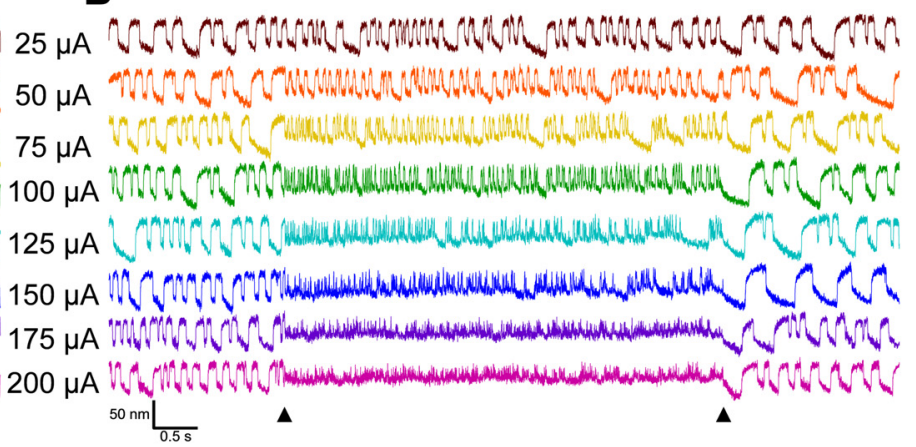

Figure 5. Increasing the stimulus amplitude for both a pulse train and a step efferent stimulus intensified the effect of the efferent stimulation. $A, A$ pulse train was applied, with $3 \mathrm{~ms}$ "on" and 10 ms "off" time. $\boldsymbol{B}, \mathrm{A}$ continuous step stimulus was applied to the saccular nerve. For both types of stimuli, the frequency of the spontaneous oscillations increased. For the step stimulus, the oscillations were suppressed at high amplitudes of the current. The labels shown between the two panels represent the current amplitudes used in both $\boldsymbol{A}$ and $\boldsymbol{B}$.

durations ("on" time), interpulse intervals ("off" time), and amplitudes. As aforementioned, hair bundles oscillated more briskly with increasing "on" times; however, for most bundles, their oscillation frequencies declined once they began spiking (Fig. 7A). However, approximately one-third of the bundles we recorded did not exhibit spiking; instead, their oscillation frequencies plateaued with rising "on" times (Fig. $7 B$ ). Figure 6A represents the mean behavior of both of these classes. All cells showed a decrease in their oscillation amplitudes in conjunction with increasing "on" times (Fig. 6B), as observed qualitatively. The change from regular oscillations to rapid, spike-like motion is reflected by the increased occurrence of the closed-channel state, and hence lower mean opening probability (Fig. 6C). There were no observable behavioral differences between the two classes of cells for Figure 6B, C. Similarly, the plots detailing the relationship between the variable "off" time with the measured bundle oscillation frequency and oscillation amplitude (Fig. $6 D, E$ ) predictably followed the visible trends shown in Figures $4 C$ and $5 A$. The mean opening probability was very weakly affected by variations in the "off" time of the stimulus train (Fig. $6 \mathrm{~F}$ ). The effect of increasing current amplitude was to enhance the frequency (Fig. $6 \mathrm{G}$ ) and decrease the amplitude of the bundle oscillation (Fig. $6 \mathrm{H}$ ); the impact on mean opening probability was similarly weak (Fig. 6I). The data in Figure $6 A-C$, Figure $6 D-F$, and Figure $6 G-I$ were obtained by averaging over 10, 7, and 9 bundles, respectively.

Our results from varying the stimulus parameters are consistent with the hypothesis that efferents exert their effect by 

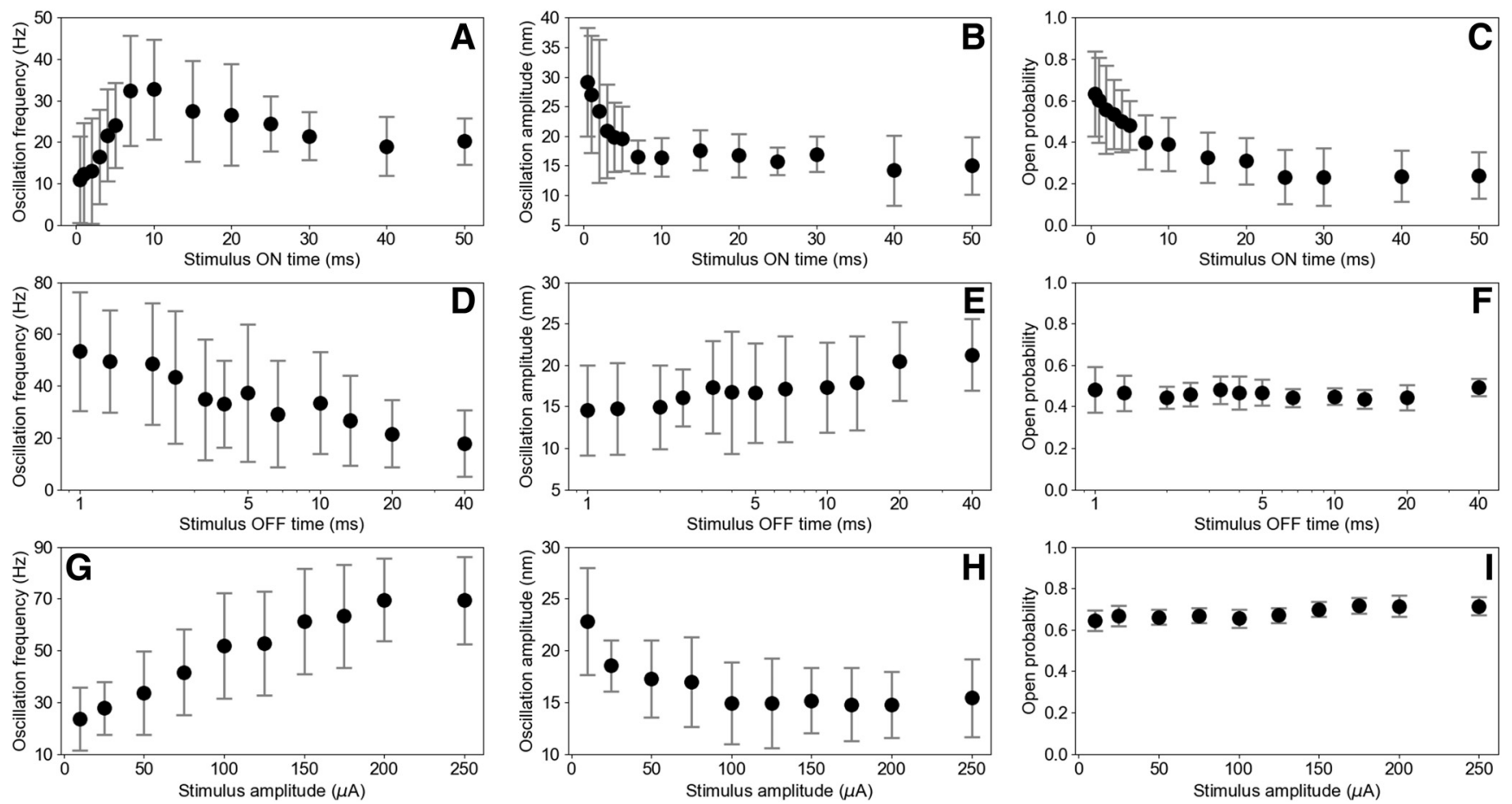

Figure 6. Varying the parameters of a pulse train stimulus affected a hair bundle's oscillation frequency, amplitude, and open probability. $A-C$, Increasing the "on" time from $0.5 \mathrm{~ms}$ up to $50 \mathrm{~ms}$ led to a decrease in both the oscillation amplitude and the open probability. Averaging the behavior of both types of bundles seen in Figure 7 produces a peak in the oscillation frequency with a softened falloff. $\boldsymbol{D}-\boldsymbol{F}$, Decreasing the "off" time from 40 to $1 \mathrm{~ms}$ led to an increase in the oscillation frequency. Similarly, the oscillation amplitude declined, whereas the open probability was fairly unaffected. G-I, Predictably, increasing the amplitude of the pulse train raised the oscillation frequency and reduced the oscillation amplitude. However, the open probability remained relatively constant. $\boldsymbol{A}-\boldsymbol{C}$ are the results of averaging the data from 10 bundles ( 2 sacculi). Similarly, $\boldsymbol{D}-\boldsymbol{F}$ were derived with data from 7 bundles ( 2 sacculi) and 9 bundles ( 2 sacculi) for $\mathbf{G}-\boldsymbol{I}$. Error bars were computed from the SDs of the measurements of individual hair bundles.
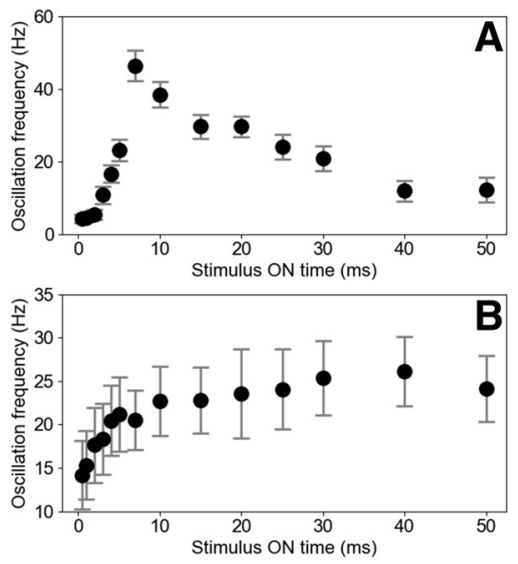

Figure 7. Incrementally changing a pulse train's "on" time resulted in two categories of hair bundle responses. In both categories, increasing the pulse duration initially elevated the bundle's oscillation frequency. Afterward, some bundles experienced a peak in their oscillation frequency, followed by a frequency decline corresponding to the exhibition of spiking behavior $(\boldsymbol{A})$. The other group of bundles displayed a plateau in their oscillation frequency $(\boldsymbol{B})$. Error bars were calculated in the same manner described for the individual hair bundles in Figure $2 C$.

hyperpolarizing the hair cell. As a control, we applied efferent stimulation in the presence of apamin, a known blocker of the SK2 channels, which was introduced into the perilymph solution. Before the apamin was introduced, the hair bundles oscillated, on average, $7.42 \pm 5.81 \mathrm{~Hz}$ (one-tailed paired $t$ test, $t_{(9)}=4.04, p=0.0015$ ) more quickly under efferent stimulation. After the addition of apamin into the perilymph, the difference in oscillation frequency during efferent modulation was $0.17 \pm 0.54 \mathrm{~Hz}$ (two-tailed paired $t$ test, $t_{(9)}=0.98, p=$ $0.35)$. The blocker hence eliminated the effects of efferent activity (Fig. 8).

Efferent activity leads to hair bundle desensitization

We next explored how efferent modulation changes the mechanical properties of a hair cell by observing how a hair bundle responds to a mechanical stimulus both with and without simultaneous efferent stimulation. In conjunction with the efferent stimulation described in the previous sections, we provided a mechanical stimulus to the hair bundle through a flexible glass probe. As evident from Figure 9, the bundle's motions are significantly less phase-locked by the mechanical stimulus in the presence of efferent stimulation. To fully explore this phenomenon, we applied a drive to the hair bundle at frequencies $3-50 \mathrm{~Hz}$, sampled in $2 \mathrm{~Hz}$ increments, with amplitudes (measured as displacements at the base of the probe) of $4,8,12,16,20,30,40,50$, and $80 \mathrm{~nm}$. Each frequency and amplitude pair was presented for 10 cycles. The first panel of Figure $10 \mathrm{~A}$ illustrates the synchronization dynamics of a hair bundle, without any efferent modulation, in the form of a receptive field map. At low mechanical stimulus amplitudes, the hair bundle showed entrainment only over a limited frequency range. However, the extent of the phaselocking grew with mechanical stimulus amplitude and created a triangular formation seen in the graph.

The hair bundle's receptive field map was drastically altered by the application of a pulse train efferent stimulus with amplitudes of 100 and $200 \mu \mathrm{A}$, shown in the second and third panels, respectively, of Figure 10A. As these two panels demonstrate, the hair bundle showed much weaker phase-locking than it had in the absence of an efferent stimulus and only did so for the highest 

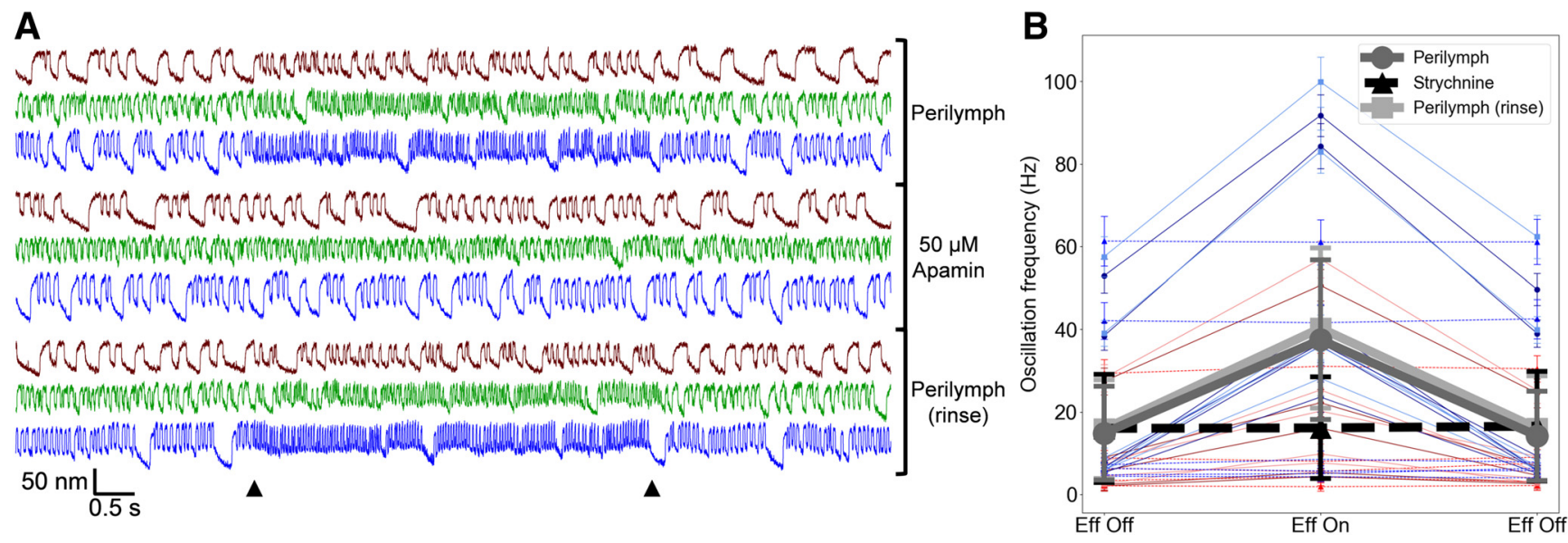

Figure 8. Apamin, a SK2 channel blocker, nullified the effects of efferent stimulation when added into the perilymph bath solution. The control was performed for a pulse train stimulus ( $\boldsymbol{A}$,

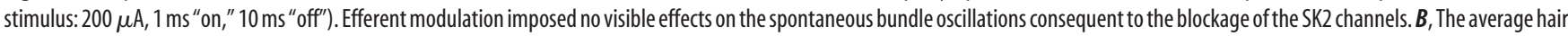
bundle oscillation frequency did not change upon efferent activity when apamin was incorporated into the basal bath solution. Circles, triangles, and squares represent oscillation frequencies in standard perilymph, apamin perilymph, and standard perilymph (rinse), respectively. $\boldsymbol{B}$ was averaged from 10 bundles ( 3 sacculi). Hair bundles originating from the same sacculus were plotted in shades of the same color. Error bars indicate the SDs of individual hair bundle oscillation frequencies.

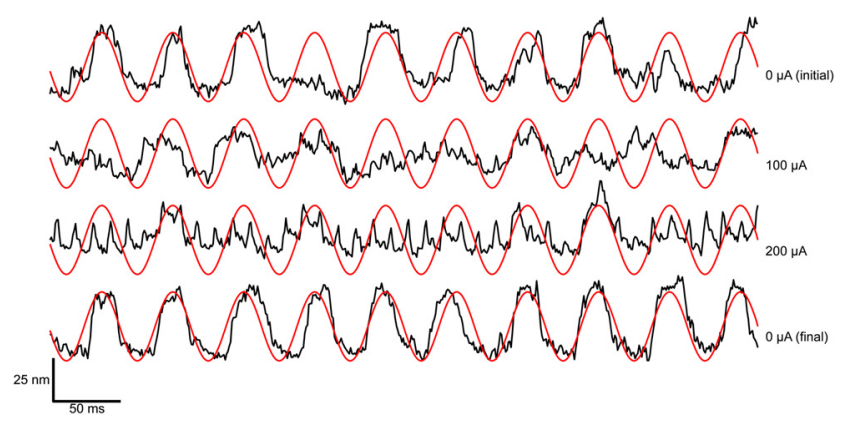

Figure 9. A hair bundle's response to sinusoidal mechanical stimuli differed greatly depending on the presence and level of efferent modulation (stimulus: $1 \mathrm{~ms}$ "on," $10 \mathrm{~ms}$ "off"). The bundle visibly phase-locked with the mechanical stimulus in the top and bottom traces. This characteristic is lost with the introduction of efferent stimulation. All four traces were recorded from the same hair bundle. The scale bars are applicable to both the bundle traces (black) and the stimulus (red).

stimulus amplitudes. Although some synchronization still occurred, the phase-locked region dramatically decreased, indicating that the hair bundle became significantly less sensitive while the efferents were activated. After the efferent stimulus was switched off, the hair bundle sensitivity showed a full return, exhibiting its original mechanical response. The sensitivity curves in Figure $10 B, C$ further demonstrate that efferent modulation mechanically desensitizes a hair cell.

We repeated the measurements, with a step stimulus substituted for the pulse train (Fig. 11). As a step stimulus exerts a stronger effect than a pulse train (equivalent to $0 \mathrm{~ms}$ "off" time), the efferent stimulus was set to amplitudes of 50 and $100 \mu \mathrm{A}$. The results are shown in the second and third panels, respectively, of Figure 11A. At $50 \mu \mathrm{A}$, the phase-locked region of the bundle's receptive field map was considerably reduced; and at $100 \mu \mathrm{A}$, the bundle exhibited only weak entrainment and showed no frequency selectivity. This supports an increased effect on the hair bundle with increasing efferent stimulus level. Moreover, there was a discernible difference in the sensitivity curves (Fig. 11 B,C) between the two levels of efferent modulation. These two figures reveal that a hair bundle's mechanical sensitivity diminishes with increasing amplitude of a step current applied to the efferent neurons.
The stated observations for pulse train and step stimuli were noted across eight hair bundles. By shifting the $x$ axis to reflect the amount of detuning from the bundle's characteristic frequency, we were able to capture the mean effect of efferent activity on a hair bundle's mechanical responsiveness. The visualization of this mean effect in Figure 12 mirrors the findings attained from individual bundles. Figure $12 A, B$ were each averaged over four bundles, with each bundle originating from a different sacculus. Each bundle was subjected to only one of the two forms of efferent stimulation. The averaged sensitivity curves (Fig. 12C,D) obtained from the receptive field maps confirmed the observed sensitivity reduction during efferent stimulation.

\section{Discussion}

Efferent impact on bundle mechanics is consistent with hyperpolarization of the hair cell soma

The physiology of efferent neurons has been widely explored in prior studies. A subset of the efferents synapse directly onto hair cells, releasing the neurotransmitter ACh and stimulating the $\alpha 9 \alpha 10$ nicotinic ACh receptors. The resulting cascade of opening ion channels in the cell soma induces changes in the membrane potential (Wersinger and Fuchs, 2011). While the ramifications of these channel openings lead to a complex range of behaviors, efferent activity is inhibitory for most of the stimuli applied (Llinás and Precht, 1969; Rossi et al., 1980; Furukawa, 1981; Ashmore and Russell, 1982; Prigioni et al., 1983; Valli et al., 1986; Sugai et al., 1991) and generally leads to hyperpolarization of the hair cell soma (Art et al., 1984; Goutman et al., 2005). In one study, hyperpolarization of the membrane potential in response to efferent stimulation was demonstrated on hair cells from the bullfrog sacculus (Castellano-Muñoz et al., 2010). Repeated pulses of efferent stimulus intensified the degree of hyperpolarization up to a saturating level. These electrophysiological data are in accordance with the influx of $\mathrm{Ca}^{2+}$ current through cholinergic receptors and a subsequent outward $\mathrm{K}^{+}$current through SK2 channels. The hyperpolarization of the soma in response to efferent actuation seems to be consistent across a number of species studied (Art et al., 1984; Wersinger and Fuchs, 2011). Concurrent with hyperpolarization, stimulation of the efferent axons was shown to generate both a reduction in sensitivity to tones at the characteristic frequency and a loss of frequency selectivity, as 
A

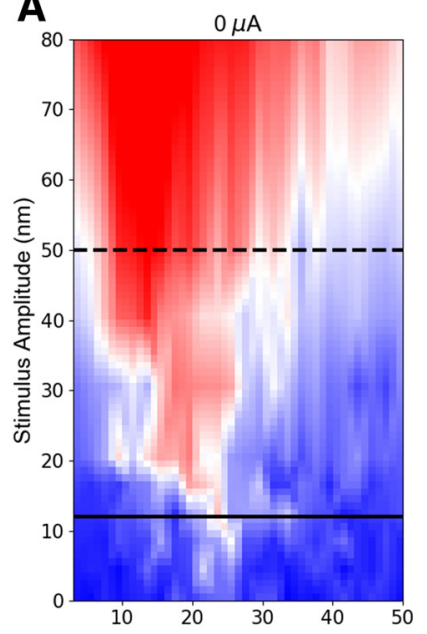

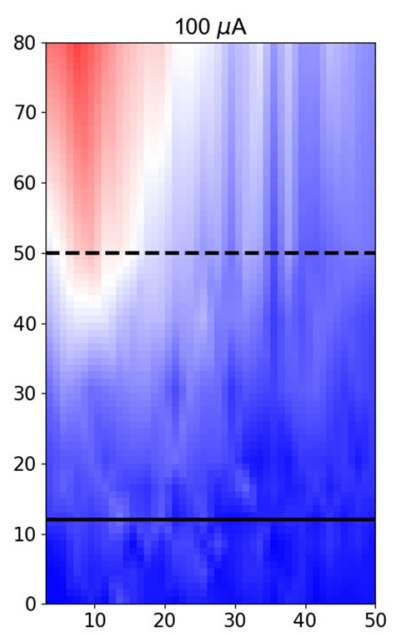
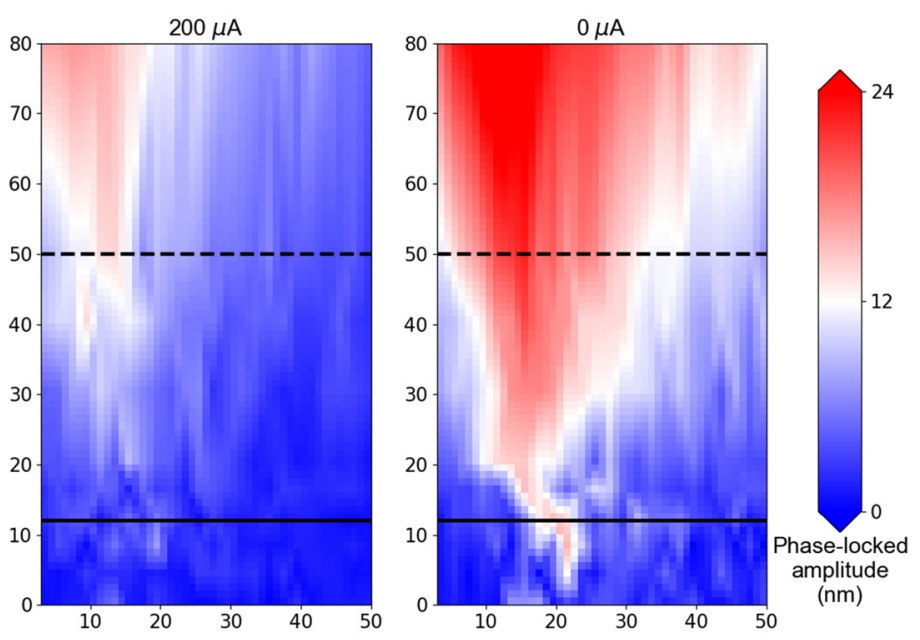

Frequency $(\mathrm{Hz})$
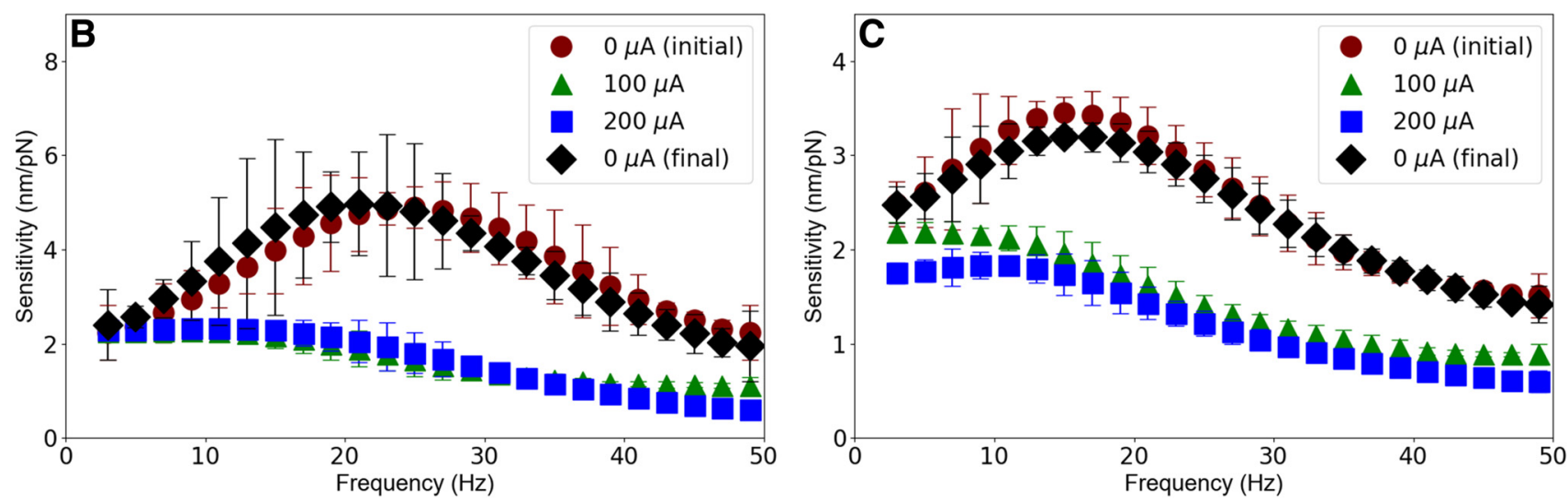

Figure 10. Receptive field maps of a hair cell were obtained with and without efferent stimulation. $\boldsymbol{A}$, Each plot was constructed by mechanically driving the bundle at 24 frequencies $(3-50 \mathrm{~Hz}$ in $2 \mathrm{~Hz}$ increments) with 10 mechanical stimulus amplitudes $(0,4,8,12,16,20,30,40,50$, and $80 \mathrm{~nm})$. The first and last panels represent measurements obtained in the absence of efferent stimulation. The second and third panels represent the mechanical response of the bundle measured while the efferent neurons were stimulated with pulse trains ( $3 \mathrm{~ms}$ " on" $/ 10 \mathrm{~ms}$ " $0 \mathrm{ff}$ ") of 100 and $200 \mu \mathrm{A}$ amplitudes, respectively. Each combination of mechanical stimulus frequency and amplitude was presented for 10 cycles. Linear interpolation was applied along the stimulus amplitude direction to yield the color maps. The bundle shows decreased phase-locking and frequency selectivity with efferent stimulation. The sensitivity curves at glass probe base displacements of ( $\boldsymbol{B}$ ) 12 $\mathrm{nm}$ (corresponding to the solid lines in $\boldsymbol{A}$ ) and $(\boldsymbol{C}) 50 \mathrm{~nm}$ (corresponding to the dashed lines in $\boldsymbol{A}$ ) demonstrate that the cell's sensitivity decreased upon efferent actuation. Circles, triangles, squares, and diamonds represent sensitivity curves with efferent stimulus amplitudes of 0 (initial), 100, 200, and $0 \mu \mathrm{A}$ (final), respectively. The sensitivity curves were smoothed by applying a running average of three data points. Error bars indicate the SDs from these means.

measured by the evoked response in afferent neurons and the somatic voltage (Art et al., 1982; Art and Fettiplace, 1984).

It is important to note that the other subset of efferents target the afferent terminals of hair cells. The stimulus protocol activates both of these efferent types, as well as afferent neurons. However, there is no known retrograde effect of afferents on hair cell activity. Hence, we assume purely efferent effects on the observed phenomena.

It is not fully understood how changes in the membrane potential of the hair cell affect active bundle mechanics. However, a number of studies have established that such an effect exists. Transepithelial electrical stimulation was shown to trigger and entrain active bundle motility (Bozovic and Hudspeth, 2003). A combination of cell electrophysiology and mechanical bundle manipulation likewise demonstrated that membrane potential impacts active motility (Meenderink et al., 2015). Steady-state changes in the membrane potential were found to modulate the frequency, amplitude, and shape of spontaneous oscillations. Specifically, hyperpolarizing a hair cell evoked an increase in the spontaneous oscillation fre- quency of the hair bundle, a decrease in the amplitude, and a reduced mean opening probability.

The repercussions on hair bundle mechanics that we observed with efferent stimulation are hence consistent with these prior studies. Efferent modulation typically raised the frequency and reduced the amplitude of spontaneous oscillations, concurring with the effect of hyperpolarizing the cell electrophysiologically. Moreover, the shape of the oscillation changed in a manner consistent with hyperpolarization, with bundles spending increasing fractions of time in the channelclosed state. This response was quantified for different levels of efferent stimulation and found to exhibit similar dependencies to those observed with increasing hyperpolarization of the hair cell. These parallels are compatible with the correlation between efferent activity and modulation of the membrane potential of the hair cell soma.

Furthermore, hair bundles became mechanically desensitized and their frequency selectivity vanished. We hypothesize that these observations are a result of the efferents shifting the hair bundle dynamics to a low-sensitivity spiking regimen, wherein 

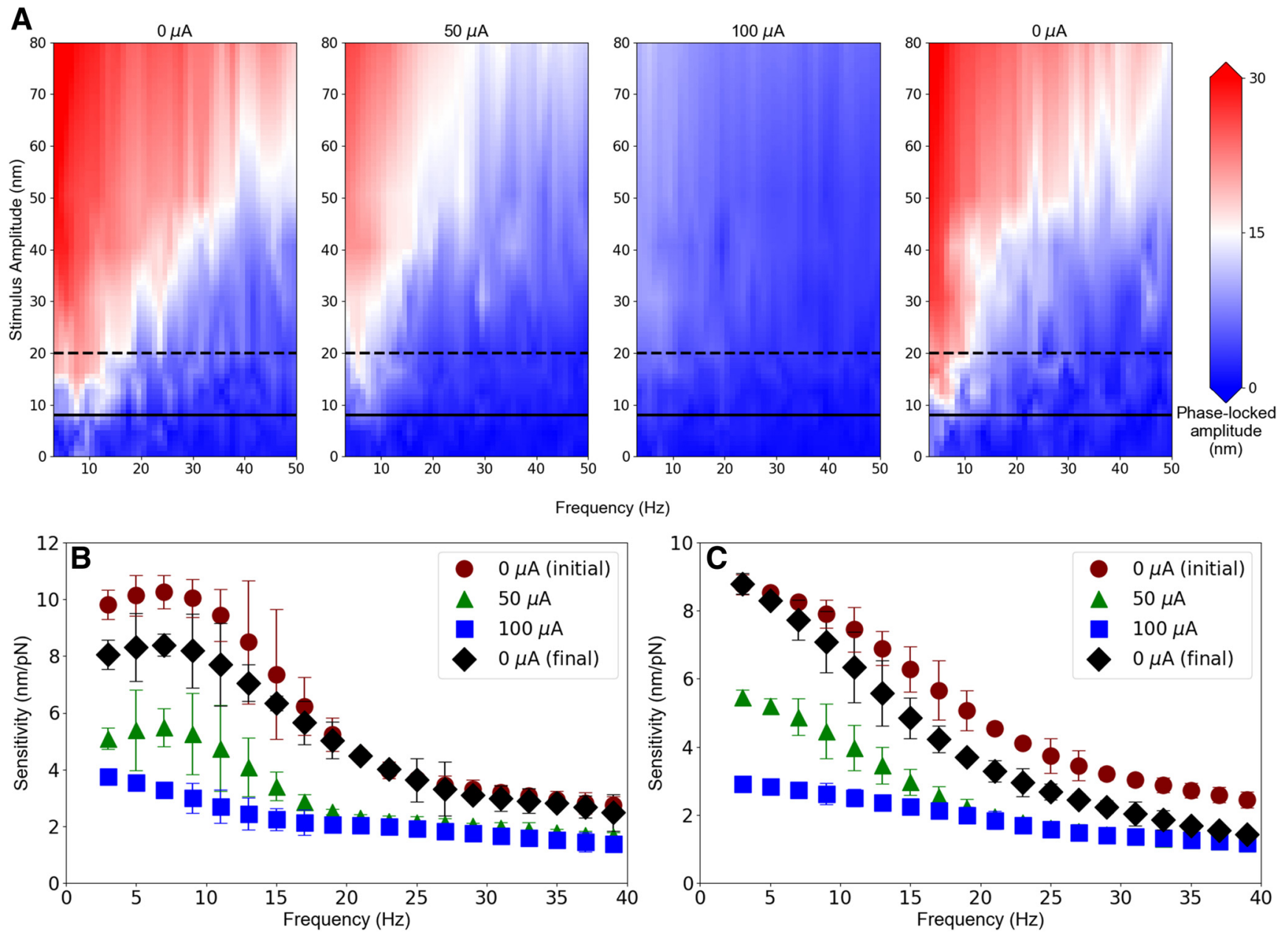

Figure 11. Receptive field maps of a hair cell were measured for varying current step amplitudes $(50,100 \mu \mathrm{A})$ of efferent stimulation $(\boldsymbol{A})$. $\boldsymbol{A}$, Each plot was constructed by mechanically driving the bundle at 24 frequencies ( $3-50 \mathrm{~Hz}$ in $2 \mathrm{~Hz}$ increments) with 10 mechanical stimulus amplitudes $(0,4,8,12,16,20,30,40$, 50 , and $80 \mathrm{~nm})$. Linear interpolation was applied along the stimulus amplitude direction. As with the pulse train stimulus, the hair bundle showed reduced phase-locking with efferent modulation. The sensitivity curves at glass probe base displacements of $(\boldsymbol{B}) 8 \mathrm{~nm}$ (corresponding to the solid lines in $\boldsymbol{A}$ ) and $(\boldsymbol{C}) 20 \mathrm{~nm}$ (corresponding to the dashed lines in $\boldsymbol{A}$ ) show that the cell became desensitized with efferent activity. Circles, triangles, squares, and diamonds represent sensitivity curves with efferent stimulus amplitudes of 0 (initial), 50,100 , and $0 \mu \mathrm{A}$ (final), respectively. The sensitivity curves were smoothed by applying a running average of three data points. Error bars indicate the SDs from these means.

the bundles mostly do not entrain in a frequency-dependent manner (Fredrickson-Hemsing et al., 2012). Although our experiments focused on the efferent impact manifested by individual bundles, we note that in vivo many species exhibit mechanical coupling from overlying structures, as well as efferent coupling from divergent innervation patterns, with each neuron synapsing onto multiple hair cells. Hence, activation of one efferent fiber is likely to have an inhibitory effect on many hair cells in parallel. In the presence of mechanical coupling between cells, there may exist a regimen in which the efferents improve the sensitivity of the system. This is a possible avenue for future study.

\section{The efferent system provides a biological mechanism for controlling the mechanical sensitivity of hair bundles}

Hair bundles exhibit remarkable sensitivity, which can be at least partly explained by several characteristics of its mechanics. First, its response is highly nonlinear, with a power-law dependence on the applied stimulus (Eguíluz et al., 2000; Hudspeth et al., 2000; Martin et al., 2000; Martin and Hudspeth, 2001). This feature has been measured in vitro and is consistent with in vivo nonlinearities observed across many species (Maoiléidigh and Ricci, 2019). Second, the hair bundle response contains internal active mech- anisms (Martin et al., 2001; Ricci et al., 2002; Nadrowski et al., 2004). In certain species, the bundles can even exhibit innate oscillations, driven by internal ATP-consuming processes (Maoiléidigh and Ricci, 2019). These spontaneous oscillations are phase-locked by an applied stimulus of much smaller amplitude, leading to an amplified response (Martin and Hudspeth, 1999; Martin et al., 2000).

There are many indications that the efferent pathway could serve as a complex gain control mechanism for both hair cells and hearing. Studies performed on the mammalian auditory system have shown that the olivocochlear efferent system plays an important role in maintaining the integrity of hearing and in finetuning its detection properties (Smith and Keil, 2015; Guinan, 2018; Lopez-Poveda, 2018). Prior work has shown that efferent activity reduces the voltage-dependent tuning of the hair cell soma (Art et al., 1982). Our study adds the finding that bundle mechanics likewise show a reduction in tuning, suggesting that these could be different manifestations of the same phenomenon. Furthermore, despite obvious physiological differences, the otoprotective role of efferents seems to be consistent across the species measured. Additionally, prior electrophysiological studies of hair cells and efferent neurons performed in vitro have shown 

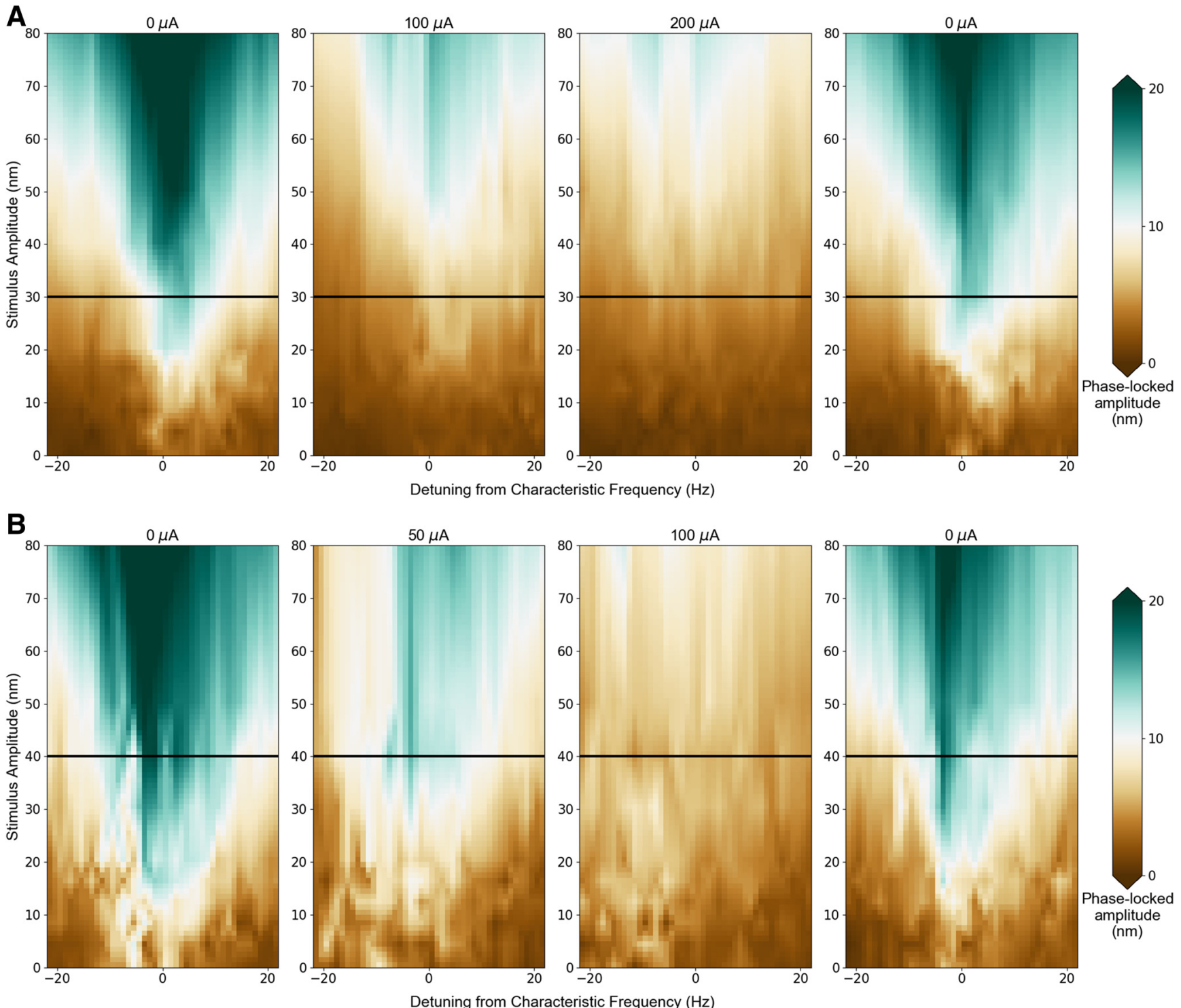

C

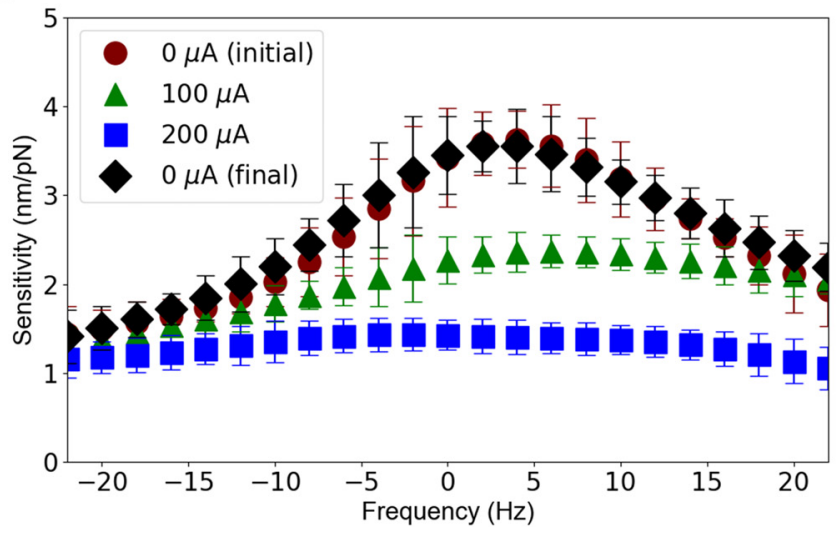

D

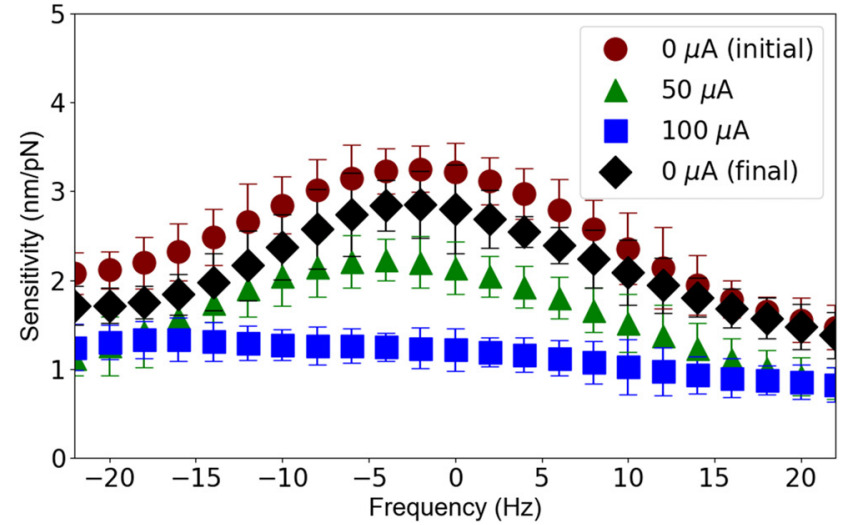

Figure 12. The receptive field maps in Figures 9 and 10 were offset along the $x$ axis to represent the detuning relative to a hair bundle's characteristic frequency. These modified maps were averaged and plotted for both pulse train $(\boldsymbol{A})$ and step $(\boldsymbol{B})$ stimuli. The characteristic frequency of each hair bundle was determined from its spontaneous oscillations. $\boldsymbol{A}, \boldsymbol{B}$ were averaged from 4 bundles each, coming from 8 sacculi. $C, D$, Averaged sensitivity curves at glass probe base displacements of 30 and $40 \mathrm{~nm}$, respectively, corresponding to the solid lines in $\boldsymbol{A}$ and $\boldsymbol{B}$, respectively. Circles, triangles, squares, and diamonds represent averaged sensitivity curves with efferent stimulus amplitudes of 0 (initial), 100,200 , and $0 \mu \mathrm{A}$ (final), respectively, for $\boldsymbol{C}$, and 0 (initial), 50,100, and $0 \mu \mathrm{A}$ (final), respectively, for $\boldsymbol{D}$. The sensitivity curves were smoothed by applying a running average of three data points. Error bars indicate the SDs from the means. 
that efference modulates the somatic membrane potential. This effect has also been seen in different end organs, indicating that some aspects of the efferent pathway are consistent across the species.

In the current study, we demonstrated that the activation of efferent neurons leads to changes in mechanical responsiveness at the level of the hair bundle. Visible changes in the innate motility of the bundle showed that its internal active processes are significantly impacted. Further, the sensitivity of the mechanical response, characterized across a range of stimulus frequencies and amplitudes, was drastically reduced. This reduction in the evoked motility of the bundle could be an important contribution to the mechanisms that underlie the otoprotective capabilities of the efferent architecture. The efferent system may therefore be viewed as a biological control mechanism that tunes the mechanical responsiveness of the hair bundle.

\section{References}

Andéol G, Guillaume A, Micheyl C, Savel S, Pellieux L, Moulin A (2011) Auditory efferents facilitate sound localization in noise in humans. J Neurosci 31:6759-6763.

Art JJ, Fettiplace R (1984) Efferent desensitization of auditory nerve fibre responses in the cochlea of the turtle pseudemys scripta elegans. J Physiol 356:507-523.

Art JJ, Crawford AC, Fettiplace R, Fuchs PA (1982) Efferent regulation of hair cells in the turtle cochlea. Proc R Soc Lond B Biol Sci 216:377-384.

Art JJ, Fettiplace R, Fuchs PA (1984) Synaptic hyperpolarization and inhibition of turtle cochlear hair cells. J Physiol 356:525-550.

Ashmore J, Russell I (1982) Effect of efferent nerve-stimulation on hair-cells of the frog sacculus. J Physiol Lond 329:25-26.

Ballestero J, Zorrilla de San Martín J, Goutman J, Elgoyhen AB, Fuchs PA, Katz E (2011) Short-term synaptic plasticity regulates the level of olivocochlear inhibition to auditory hair cells. J Neurosci 31:14763-14774.

Blanchet C, Eróstegui C, Sugasawa M, Dulon D (1996) Acetylcholineinduced potassium current of guinea pig outer hair cells: its dependence on a calcium influx through nicotinic-like receptors. J Neurosci 16:25742584.

Boero LE, Castagna VC, Di Guilmi MN, Goutman JD, Elgoyhen AB, GómezCasati ME (2018) Enhancement of the medial olivocochlear system prevents hidden hearing loss. J Neurosci 38:7440-7451.

Bozovic D, Hudspeth AJ (2003) Hair-bundle movements elicited by transepithelial electrical stimulation of hair cells in the sacculus of the bullfrog. Proc Natl Acad Sci U S A 100:958-963.

Castellano-Muñoz M, Israel SH, Hudspeth AJ (2010) Efferent control of the electrical and mechanical properties of hair cells in the bullfrog's sacculus. PLoS One 5:e13777.

Cooper NP, Guinan JJ Jr (2006) Efferent-mediated control of basilar membrane motion. J Physiol 576:49-54.

Darrow KN, Maison SF, Liberman MC (2006) Cochlear efferent feedback balances interaural sensitivity. Nat Neurosci 9:1474-1476.

Eguíluz VM, Ospeck M, Choe Y, Hudspeth AJ, Magnasco MO (2000) Essential nonlinearities in hearing. Phys Rev Lett 84:5232-5235.

Fredrickson-Hemsing L, Ji S, Bruinsma R, Bozovic D (2012) Mode-locking dynamics of hair cells of the inner ear. Phys Rev E Stat Nonlin Soft Matter Phys 86:021915.

Fuchs P (2002) The synaptic physiology of cochlear hair cells. Audiol Neurotol 7:40-44.

Fuchs PA, Murrow B (1992) A novel cholinergic receptor mediates inhibition of chick cochlear hair cells. Proc Biol Sci 248:35-40.

Furukawa T (1981) Effects of efferent stimulation on the saccule of goldfish. J Physiol 315:203-215.

Giraud AL, Garnier S, Micheyl C, Lina G, Chays A, Chéry-Croze S (1997) Auditory efferents involved in speech-in-noise intelligibility. Neuroreport 8:1779-1783.

Gómez-Casati ME, Fuchs PA, Elgoyhen AB, Katz E (2005) Biophysical and pharmacological characterization of nicotinic cholinergic receptors in rat cochlear inner hair cells. J Physiol 566:103-118.

Goutman JD, Fuchs PA, Glowatzki E (2005) Facilitating efferent inhibition of inner hair cells in the cochlea of the neonatal rat. J Physiol 566:49-59.
Guinan JJ (2011) Physiology of the medial and lateral olivocochlear systems. In: Auditory and vestibular efferents, pp 39-81. New York: Springer.

Guinan JJ Jr (2018) Olivocochlear efferents: their action, effects, measurement and uses, and the impact of the new conception of cochlear mechanical responses. Hear Res 362:38-47.

Guth PS, Dunn A, Kronomer K, Norris CH (1994) The cholinergic pharmacology of the frog saccule. Hear Res 75:225-232.

Handrock M, Zeisberg J (1982) The influence of the efferent system on adaptation, temporary and permanent threshold shift. Arch Otorhinolaryngol 234:191-195.

Hartigan JA, Hartigan PM (1985) The dip test of unimodality. Ann Stat 13: $70-84$.

Highstein SM (1991) The central nervous system efferent control of the organs of balance and equilibrium. Neurosci Res 12:13-30.

Highstein SM, Baker R (1985) Action of the efferent vestibular system on primary afferents in the toadfish, Opsanus tau. J Neurophysiol 54:370384.

Howard J, Hudspeth AJ (1987) Mechanical relaxation of the hair bundle mediates adaptation in mechanoelectrical transduction by the bullfrog's saccular hair cell. Proc Natl Acad Sci U S A 84:3064-3068.

Hudspeth AJ (2014) Integrating the active process of hair cells with cochlear function. Nat Rev Neurosci 15:600-614.

Hudspeth AJ (1983) The hair cells of the inner ear. Sci Am 248:54-64.

Hudspeth AJ, Choe Y, Mehta AD, Martin P (2000) Putting ion channels to work: mechanoelectrical transduction, adaptation, and amplification by hair cells. Proc Natl Acad Sci U S A 97:11765-11772.

Jamali M, Sadeghi SG, Cullen KE (2009) Response of vestibular nerve afferents innervating utricle and saccule during passive and active translations. J Neurophysiol 101:141-149.

Kim S, Frisina RD, Frisina DR (2006) Effects of age on speech understanding in normal hearing listeners: relationship between the auditory efferent system and speech intelligibility in noise. Speech Commun 48:855-862.

Kong JH, Adelman JP, Fuchs PA (2008) Expression of the sk2 calciumactivated potassium channel is required for cholinergic function in mouse cochlear hair cells. J Physiol 586:5471-5485.

Kujawa SG, Liberman MC (2009) Adding insult to injury: cochlear nerve degeneration after "temporary" noise-induced hearing loss. J Neurosci 29:14077-14085.

Kumar UA, Vanaja CS (2004) Functioning of olivocochlear bundle and speech perception in noise. Ear Hear 25:142-146.

Llinás R, Precht W (1969) The inhibitory vestibular efferent system and its relation to the cerebellum in the frog. Exp Brain Res 9:16-29.

Lopez-Poveda EA (2018) Olivocochlear efferents in animals and humans: from anatomy to clinical relevance. Front Neurol 9:197.

Lopez-Poveda EA, Eustaquio-Martín A, Stohl JS, Wolford RD, Schatzer R, Wilson BS (2016) Roles of the contralateral efferent reflex in hearing demonstrated with cochlear implants. In: Physiology, psychoacoustics and cognition in normal and impaired hearing, pp 105-114. New York: Springer.

Maison SF, Liberman MC (2000) Predicting vulnerability to acoustic injury with a noninvasive assay of olivocochlear reflex strength. J Neurosci 20: 4701-4707.

Maison SF, Usubuchi H, Liberman MC (2013) Efferent feedback minimizes cochlear neuropathy from moderate noise exposure. J Neurosci 33:55425552 .

Maoiléidigh DÓ, Ricci AJ (2019) A bundle of mechanisms: Inner-ear haircell mechanotransduction. Trends Neurosci 42:221-236.

Martin P, Hudspeth AJ (1999) Active hair-bundle movements can amplify a hair cell's response to oscillatory mechanical stimuli. Proc Natl Acad Sci U S A 96:14306-14311.

Martin P, Hudspeth AJ (2001) Compressive nonlinearity in the hair bundle's active response to mechanical stimulation. Proc Natl Acad Sci U S A 98:14386-14391.

Martin P, Mehta AD, Hudspeth AJ (2000) Negative hair-bundle stiffness betrays a mechanism for mechanical amplification by the hair cell. Proc Natl Acad Sci U S A 97:12026-12031.

Martin P, Hudspeth AJ, Jülicher F (2001) Comparison of a hair bundle's spontaneous oscillations with its response to mechanical stimulation reveals the underlying active process. Proc Natl Acad Sci U S A 98: $14380-14385$.

Meenderink SW, Quiñones PM, Bozovic D (2015) Voltage-mediated con- 
trol of spontaneous bundle oscillations in saccular hair cells. J Neurosci 35:14457-14466.

Micheyl C, Collet L (1996) Involvement of the olivocochlear bundle in the detection of tones in noise. J Acoust Soc Am 99:1604-1610.

Micheyl C, Perrot X, Collet L (1997) Relationship between auditory intensity discrimination in noise and olivocochlear efferent system activity in humans. Behav Neurosci 111:801-807.

Murugasu E, Russell IJ (1996) The effect of efferent stimulation on basilar membrane displacement in the basal turn of the guinea pig cochlea. J Neurosci 16:325-332.

Nadrowski B, Martin P, Jülicher F (2004) Active hair-bundle motility harnesses noise to operate near an optimum of mechanosensitivity. Proc Natl Acad Sci U S A 101:12195-12200.

Oliver D, Klöcker N, Schuck J, Baukrowitz T, Ruppersberg JP, Fakler B (2000) Gating of $\mathrm{Ca}^{2+}$-activated $\mathrm{K}^{+}$channels controls fast inhibitory synaptic transmission at auditory outer hair cells. Neuron 26:595-601.

Prigioni I, Valli P, Casella C (1983) Peripheral organization of the vestibular efferent system in the frog: an electrophysiological study. Brain Res 269:83-90.

Rabbitt RD, Brownell WE (2011) Efferent modulation of hair cell function. Curr Opin Otolaryngol Head Neck Surg 19:376-381.

Ramunno-Johnson D, Strimbu CE, Fredrickson L, Arisaka K, Bozovic D (2009) Distribution of frequencies of spontaneous oscillations in hair cells of the bullfrog sacculus. Biophys J 96:1159-1168.

Reiter ER, Liberman MC (1995) Efferent-mediated protection from acoustic overexposure: relation to slow effects of olivocochlear stimulation. J Neurophysiol 73:506-514.

Ricci AJ, Crawford AC, Fettiplace R (2002) Mechanisms of active hair bundle motion in auditory hair cells. J Neurosci 22:44-52.

Robinson BL, McAlpine D (2009) Gain control mechanisms in the auditory pathway. Curr Opin Neurobiol 19:402-407.

Rohmann KN, Wersinger E, Braude JP, Pyott SJ, Fuchs PA (2015) Activation of BK and SK channels by efferent synapses on outer hair cells in high-frequency regions of the rodent cochlea. J Neurosci 35:1821-1830.

Rossi ML, Prigioni I, Valli P, Casella C (1980) Activation of the efferent system in the isolated frog labyrinth: effects on the afferent EPSPs and spike discharge recorded from single fibers of the posterior nerve. Brain Res 185:125-137.

Russell IJ, Murugasu E (1997) Medial efferent inhibition suppresses basilar membrane responses to near characteristic frequency tones of moderate to high intensities. J Acoust Soc Am 102:1734-1738.

Salvi JD, Maoiléidigh DÓ, Fabella BA, Tobin M, Hudspeth AJ (2015) Control of a hair bundle's mechanosensory function by its mechanical load. Proc Natl Acad Sci U S A 112:E1000-E1009.
Salvi JD, Maoiléidigh DÓ, Hudspeth AJ (2016) Identification of bifurcations from observations of noisy biological oscillators. Biophys J 111: $798-812$.

Seebacher J, Franke-Trieger A, Weichbold V, Zorowka P, Stephan K (2019) Improved interaural timing of acoustic nerve stimulation affects sound localization in single-sided deaf cochlear implant users. Hear Res 371:19-27.

Smith DW, Keil A (2015) The biological role of the medial olivocochlear efferents in hearing: separating evolved function from exaptation. Front Syst Neurosci 9:12.

Sugai T, Sugitani M, Ooyama H (1991) Effects of activation of the divergent efferent fibers on the spontaneous activity of vestibular afferent fibers in the toad. Jpn J Physiol 41:217-232.

Sugai T, Yano J, Sugitani M, Ooyama H (1992) Actions of cholinergic agonists and antagonists on the efferent synapse in the frog sacculus. Hear Res 61:56-64.

Taranda J, Maison SF, Ballestero JA, Katz E, Savino J, Vetter DE, Boulter J, Liberman MC, Fuchs PA, Elgoyhen AB (2009) A point mutation in the hair cell nicotinic cholinergic receptor prolongs cochlear inhibition and enhances noise protection. PLoS Biol 7:e1000018.

Taylor P, Brown JH (1999) Synthesis, storage and release of acetylcholine. In: Basic neurochemistry: molecular, cellular and medical aspects, Ed 6. Philadelphia: Lippincott-Raven.

Valli P, Botta L, Zucca G, Casella C (1986) Functional organization of the peripheral efferent vestibular system in the frog. Brain Res 362:92-97.

Wedemeyer C, Vattino LG, Moglie MJ, Ballestero J, Maison SF, Di Guilmi MN, Taranda J, Liberman MC, Fuchs PA, Katz E, Elgoyhen AB (2018) A gain-of-function mutation in the $\alpha 9$ nicotinic acetylcholine receptor alters medial olivocochlear efferent short-term synaptic plasticity. J Neurosci 38:3939-3954

Wersinger E, Fuchs PA (2011) Modulation of hair cell efferents. Hear Res $279: 1-12$.

Westfall T (2009) Cholinergic neurotransmission in the autonomic and somatic motor nervous system. In: Encyclopedia of Neuroscience, pp 827834. Los Angeles: Academic.

Yoshida N, Liberman MC, Brown MC, Sewell WF (2001) Fast, but not slow, effects of olivocochlear activation are resistant to apamin. J Neurophysiol 85:84-88.

Zheng XY, McFadden SL, Ding DL, Henderson D (2000) Cochlear deefferentation and impulse noise-induced acoustic trauma in the chinchilla. Hear Res 144:187-195.

Zirn S, Angermeier J, ArndtS, AschendorffA, Wesarg T (2019) Reducing the device delay mismatch can improve sound localization in bimodal cochlear implant or hearing-aid users. Trends Hear 23:2331216519843876. 